\title{
Removing Piston-driven Mechanical Chest Compression Artefacts from the ECG
}

\author{
Iraia Isasi ${ }^{1}$, Unai Irusta ${ }^{1}$, Elisabete Aramendi ${ }^{1}$, Unai Ayala ${ }^{2}$, Erik Alonso $^{3}$, Jo Kramer-Johansen ${ }^{4}$, \\ Trygve Eftest $\varnothing 1^{5}$ \\ ${ }^{1}$ Communications Engineering, University of the Basque Country (UPV/EHU), Bilbao, Spain \\ ${ }^{2}$ Signal Processing and Communications, Mondragon University, Mondragon, Spain \\ ${ }^{3}$ Department of Applied Mathematics, University of the Basque Country (UPV/EHU), Bilbao, Spain \\ ${ }^{4}$ Norwegian National Advisory Unit on Prehospital Emergency Medicine, \\ Oslo University Hospital and University of Oslo, Norway \\ ${ }^{5}$ Electrical Engineering and Computer Science. University of Stavanger, Stavanger, Norway
}

\begin{abstract}
Piston-driven mechanical chest compression (CC) devices induce a quasi-periodic artefact in the ECG, making rhythm diagnosis unreliable. Data from 230 out-of-hospital cardiac arrest (OHCA) patients were collected in which CCs were delivered using the piston driven LUCAS-2 device. Underlying rhythms were annotated by expert reviewers in artefact-free intervals. Two artefact removal methods (filters) were introduced: a static solution based on Goertzel's algorithm, and an adaptive solution based on a Recursive Least Squares (RLS) filter. The filtered ECG was diagnosed by a shock/no-shock decision algorithm used in a commercial defibrillator and compared with the rhythm annotations. Filter performance was evaluated in terms of balanced accuracy (BAC), the mean of sensitivity (shockable) and specificity (nonshockable). Compared to the unfiltered signal, the static filter increased BAC by 20 points, and the RLS filter by 25 points. Adaptive filtering results in $99.0 \%$ sensitivity and $87.3 \%$ specificity.
\end{abstract}

\section{Introduction}

Early defibrillation and high-quality cardiopulmonary resuscitation (CPR) are crucial to improve chances of survival from out of hospital cardiac arrest (OHCA) [1]. Chest compressions (CCs) provided during CPR introduce artefacts in the ECG, invalidating the diagnosis of any rhythm analysis algorithm. Currently compressions are interrupted for the analysis, but these hands-off intervals compromise circulation and thus reduce the probability of restoration of spontaneous circulation (ROSC) and survival[2]. Although solutions to analyse the rhythm during pauses in $\mathrm{CC}$ exist $[3,4]$, rhythm analysis during $\mathrm{CCs}$ requires a filter to remove $\mathrm{CC}$ artefacts. Many such filters have been proposed to permit a reliable diagnosis during CCs $[5,6]$, but no effective solution has been integrated into current defibrillators yet.

Piston-driven mechanical CC devices are increasingly used in resuscitation. These devices deliver CCs with a constant rate and depth ensuring CPR is delivered according to resuscitation guidelines. Their use is especially recommended during transportation, invasive procedures or prolonged CPR. One such device is the LUCAS 2 (Physio-Control/Jolife AB, Lund, Sweden). The LUCAS 2 provides chest compressions in a fixed position, constant depth (40-53 mm depending on chest height), constant rate $\left(102 \pm 2 \mathrm{~min}^{-1}, 1.694 \mathrm{~Hz}\right), 50 \%$ duty-cycle and full chest recoil after each compression [7]. We should expect the artefact caused by LUCAS 2 to have a periodical pattern at the constant frequency of the CCs.

This study evaluates the feasibility of analyzing the rhythm during mechanical CCs provided by LUCAS 2 on OHCA data. Two artefact removal alternatives were compared: an adaptive filtering method based on a Recursive Least Square (RLS) algorithm and a non-adaptive (static) filtering method which uses Goertzel's algorithm to model the artefact.

\section{Materials and methods}

\subsection{Materials}

The data used for this study were gathered by the emergency services of Oslo and Akershus (Norway) with the LifePak 15 defibrillators (Physio-Control Inc., Redmond, WA, USA). The recorded ECG and thoracic 

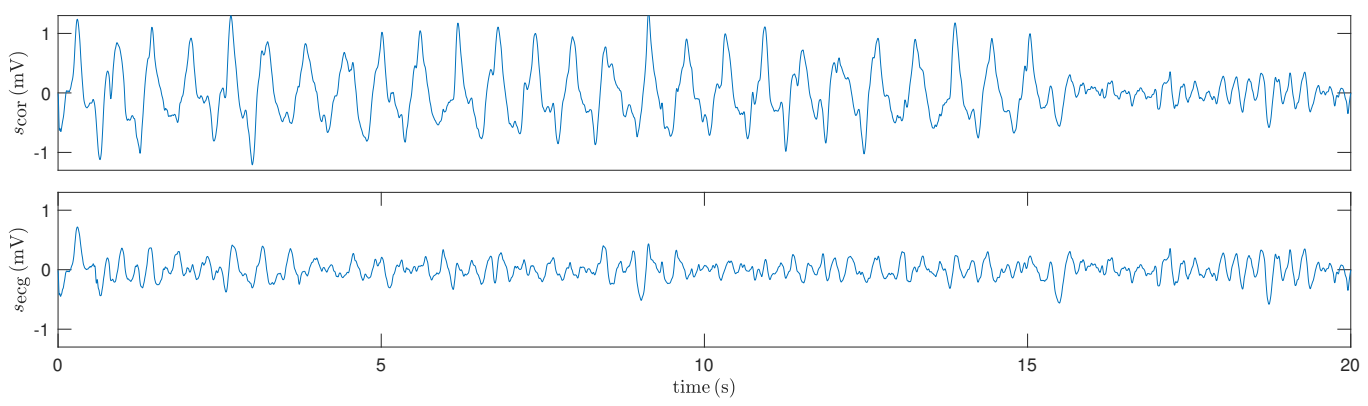

Figure 1. A 20 s episode of a patient in ventricular fibrillation (VF), before filtering (top) and after filtering (bottom). The initial $15 \mathrm{~s}$ show ECG records during CCs delivered by the LUCAS 2 (quasi-periodic artefact). The last $5 \mathrm{~s}$ show the underlying VF, in an interval without CCs. Filtering (bottom pannel) reveals the underlying rhythm.

impedance (TI) signals were exported to Matlab using the Codestat (Physio-Control Inc.) research tool, and resampled to $250 \mathrm{~Hz}$. Details on the dataset are further described in [7].

The dataset contains 1045 segments of $20 \mathrm{~s}$ from 230 patients. The first $15 \mathrm{~s}$ included continuous $\mathrm{CCs}$, the last $5 \mathrm{~s}$ were free of artefacts and were used by expert reviewers to assess the underlying rhythm. The dataset contains 201 shockable and 844 nonshockable rhythms (270 asystole, 574 organized) [7].

\subsection{Methods}

ECG segments were band-pass filtered to a typical automatic external defibrillator (AED) bandwidth, using an order 8 Butterworth filter $(0.5-40 \mathrm{~Hz})$.

\section{Model of the compression artefact}

The CC artefact in the ECG is customarily modelled as additive noise:

$$
s_{\mathrm{cor}}(n)=s_{\mathrm{ecg}}(n)+s_{\mathrm{cc}}(n)
$$

where $s_{\text {cor }}$ is the ECG corrupted by the CC artefact, $s_{\mathrm{cc}}$, and $s_{\mathrm{ecg}}$ is the ECG which reflects the underlying heart rhythm. For a piston-driven compression device the artefact, $s_{\mathrm{cc}}$, can be approximated by a (quasi)-periodic signal in term of $N$ harmonics of the fundamental frequency, $f_{0}=1.694 \mathrm{~Hz}$. Assuming a model with $N$ Fourier coefficients $c_{k}=\left|c_{k}\right| e^{j \theta_{k}}$, the artefact can be simply written as:

$$
\begin{aligned}
& s_{\mathrm{cc}}(n)=A(n) \sum_{k=1}^{N}\left|c_{k}\right| \cos \left(k \omega_{0} n T_{s}+\theta_{k}\right)= \\
& =A(n) \sum_{k=1}^{N} a_{k} \cos \left(k \omega_{0} n T_{s}\right)+b_{k} \sin \left(k \omega_{0} n T_{s}\right)
\end{aligned}
$$

where $T_{s}$ is the sampling period, $\omega_{0}=2 \pi f_{0}$ and $A(n)$ is an amplitude envelope to differentiate intervals with $(A=$ $1)$ and without compressions $(A=0)$.

The two methods proposed in this paper assume different natures for the Fourier coefficients. In the static solution, $c_{k}=\left|c_{k}\right| e^{j \theta_{k}}$ are constant over time. In the adaptive solution the coefficients are assumed to be time-varying $c_{k}(n)=\left|c_{k}(n)\right| e^{j \theta_{k}(n)}$, with small changes every sample. Once $s_{\mathrm{cc}}$ is estimated, the underlying rhythm $s_{\text {ecg }}$ is obtained by subtraction using equation (1), and then fed to a shock/no-shock decision algorithm for diagnosis.

\section{Static solution}

The static solution assumes the $N$ Fourier coefficients are constant. Since only just a few frequency components of $s_{\text {cor }}$ signal are of interest, Goertzel's algorithm can be used to estimate those spectral components instead of analyzing all frequency components of the Discrete-Time Fourier Transform (DTFT). However, since for an $L$ point signal the frequency resolution of Goertzel's algorithm is $\Delta f=f_{s} / L$, the fundamental frequency of the signal must be an integer multiple of $\Delta f$. This is not the case for $f_{0}=1.694 \mathrm{~Hz}$ (LUCAS 2), so, the Generalized Goertzel algorithm was used. This generalization allows the calculation of spectral components at any frequency, by extending the DTFT to any real frequency $\omega_{\ell}=2 \pi \ell / L$. Then the frequency component is estimated as:

$$
X\left(\omega_{\ell}\right)=e^{-j 2 \pi \ell} \sum_{n=0}^{L-1} x(n) e^{-j 2 \pi \ell \frac{n-L}{L}}
$$

to which the custom Goertzel's algorithm is applied [8]:

$$
\begin{aligned}
& s(n)=x(n)+2 \cos \left(\frac{2 \pi \ell}{N}\right) s(n-1)-s(n-2) \\
& y(n)=\left(s(n)-e^{-j \frac{2 \pi \ell}{N}} s(n-1)\right) e^{-j 2 \pi \ell}
\end{aligned}
$$

and $y(L-1)=X\left(\omega_{\ell}\right)$. In our case the signal was first windowed using a Kaiser window $w_{\beta}(n)$, to form 
$x_{w}(n)=s_{\text {cor }}(n) \cdot w_{\beta}(n)$, so the spectral component was obtained as:

$$
c_{k}=\frac{2}{W_{\beta}(0)} X_{w}\left(\omega_{\ell}\right), \quad \ell \in \mathbb{R}
$$

where $W_{\beta}$ is the Fourier transform of the Kaiser window, and $X_{w}\left(\omega_{\ell}\right)$ is the Fourier transform of $x_{w}(n)$ as shown in equation (4) and computed using Goertzel's algorithm. In the Kaiser window the form factor $\beta$ controlls the window trade-off between side-lobe level and main-lobe width. For each segment, the $c_{k}$ coefficients were estimated using an interval of $5 \mathrm{~s}$ with uninterrupted CCs.

\section{Adaptive solution}

In the adaptive solution the time-varying Fourier coefficients, $a_{k}(n), b_{k}(n)$, were estimated using an RLS filter that tracks the spectral components of the artefact [9].

The in-phase, $a_{k}(n)$, and quadrature, $b_{k}(n)$, components model the artefact as described by equation (8), which is equation (3) in vector notation:

$$
s_{\mathrm{cc}}(n)=\Theta_{n-1}^{T} \Phi_{n}
$$

where,

$$
\begin{aligned}
\Theta_{n}= & {\left[a_{1}(n) b_{1}(n) \ldots a_{N}(n) b_{N}(n)\right]^{T} } \\
\Phi_{n}= & {\left[\cos \left(\omega_{0} n T_{s}\right) \sin \left(\omega_{0} n T_{s}\right) \ldots\right.} \\
& \left.\cos \left(N \omega_{0} n T_{s}\right) \sin \left(N \omega_{0} n T_{s}\right)\right]^{T}
\end{aligned}
$$

The model of the artefact is updated through the $a_{k}(n)$ and $b_{k}(n)$ coefficients in each iteration. The filtered $s_{\text {ecg }}$ and the filter coefficients are computed as follows:

$$
\begin{aligned}
s_{\mathrm{ecg}}(n) & =s_{\mathrm{cor}}(n)-s_{\mathrm{cc}}(n) \\
\Theta_{n} & =\Theta_{n-1}+F_{n} \Phi_{n} s_{\mathrm{ecg}}(n) \\
F_{n} & =\frac{1}{\lambda}\left[F_{n-1}-\frac{F_{n-1} \Phi_{n} \Phi_{n}^{T} F_{n-1}}{\lambda+\Phi_{n}^{T} F_{n-1} \Phi_{n}}\right]
\end{aligned}
$$

where the forgetting factor $\lambda$ is usually close to one, and defines the convergence rate, the tracking power, misadjustement and stability of the RLS filter.

\subsection{Evaluation}

The ECG filtered through both methods was diagnosed by a shock/no-shock decision algorithm, the Matlab version of the algorithm designed for the Reanibex R-series defibrillators (Bexen Cardio, Ermua, Spain). This algorithm diagnoses the ECG in less than $9.6 \mathrm{~s}$ by analyzing 2 or 3 consecutive $3.2 \mathrm{~s}$ intervals of the ECG [10]. The interval from $3.4 \mathrm{~s}$ to $13 \mathrm{~s}$ of each segment was diagnosed in order to avoid filtering transients. The diagnoses were compared with the rhythm annotations to obtain the proportion of correctly classified shockable (sensitivity, SE) and nonshockable (specificity, $\mathrm{SP})$ rhythms.

Filter performance was evaluated in terms of the balanced accuracy $(\mathrm{BAC}), \mathrm{BAC}=0.5(\mathrm{SE}+\mathrm{SP})$, within the following working ranges: $10<N<30$ and $0<\beta<15$ for the static filter, and $10<N<30$ and $0.965<\lambda<0.999$ for the adaptive filter. Finally, within those ranges a 100 bootstrapped patient-wise 5-fold cross validation approach was used to obtain an estimate of the statistical distribution of SE and SP. SE/SP values will be reported as mean (CI, 95\% confidence interval).

\section{Results}

Figure 2 shows the BAC for the static (left) and adaptive (right) filters within the working ranges for three significant values of $N$. As seen in figure 2, both filters showed a working range in which the performance was close to optimal in terms of BAC. In the case of the static filter, the best results were obtained for $4<\beta<5$ and $N>20$. The range for the RLS filter was $0.989<\lambda<$ 0.993 and $N>20$. In fact, for smaller values of $N$ (see figure 2) the $\mathrm{BAC}$ in the optimal $\beta$ and $\lambda$ ranges is smaller in both cases.

Table 1 shows the bootstraped SE/SP and BAC after filtering, compared to the values obtained before filtering.

\begin{tabular}{lcccc}
\hline & & unfilt & Goertzel & RLS \\
\hline SE & $(\%)$ & 50.7 & $97.0(95.5-97.5)$ & $99.0(97.0-99.5)$ \\
SP & $(\%)$ & 83.9 & $80.2(79.5-81.0)$ & $87.3(86.5-87.6)$ \\
BAC (\%) & 67.3 & $88.6(87.8-89.3)$ & $93.0(91.9-93.5)$ \\
\hline
\end{tabular}

Table 1. Accuracy before and after filtering.

Both filters resulted in an increase of over 30 points in SE with a slight change in SP. The shock/no-shock decision after applying the adaptive filter were more accurate than those obtained after applying the static filter.

\section{Discussion}

This study introduces two different filtering techniques to remove CPR artefact from the ECG during mechanical compressions. Both methods represent the artefact as a (quasi)-periodic signal with a fundamental frequency equal to the frequency of the compressions and $N$ harmonics. Whereas the static method assumes that the artefact is periodic, the adaptive method considers slow fluctuations from cycle to cycle.

Mechanically delivered compressions have very stable frequency, depth and duty cycle. We might assume little 


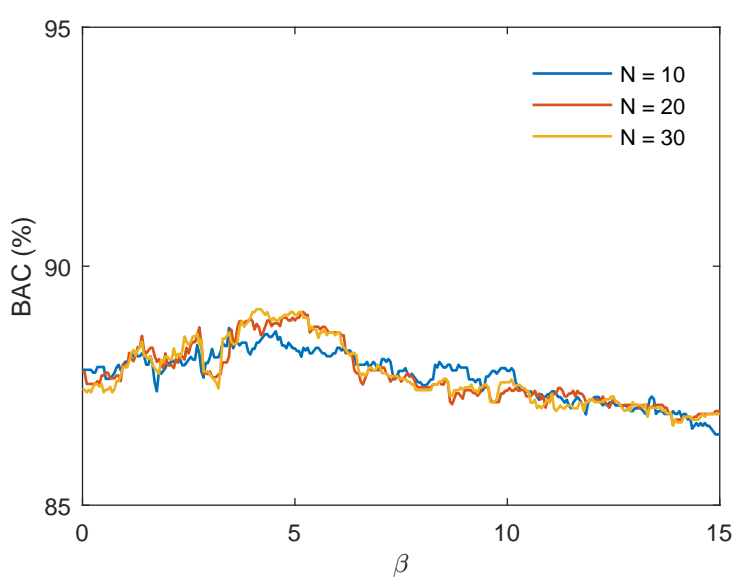

(a) Static solution

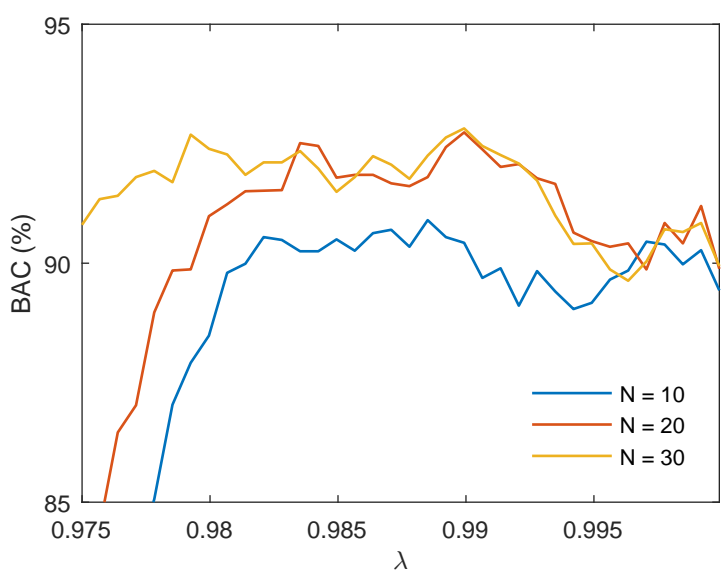

(b) Adaptive solution

Figure 2. Performance of the static and the adaptive filtering methods in terms of $N$ and $\beta$ for the Goertzel filter (panel a), and in terms of $N$ and $\lambda$ for the RLS filter (panel b).

change in the artefact from CC cycle to cycle, but the results of this study show the need of an adaptive solution. Both methods resulted in a significant increase in BAC but the RLS filter produced better results than the static solution (approximately 2 points more in SE and 7 points more in SP). The adaptive solution was able to track the small fluctuations of the artefact from cycle to cycle.

In conclusion, the results showed that the adaptive filtering provided acceptable values for an acccurate rhythm diagnosis during compressions, particularly for shockable rhythms (SE>98\%). However, further analysis is recommended to increase the accuracy, mainly, for nonshockable rhythms. The results reported in this and in previous studies [7] are still below the 95\% recommended for nonshockable rhythms by the American Heart Association.

\section{Acknowledgements}

This work received financial support from the Spanish Ministerio de Economía y Competitividad, project TEC2015-64678-R jointly with the Fondo Europeo de Desarrollo Regional (FEDER); from UPV/EHU via project 323616NCAU and from the Basque Government through grant PRE-2016-1-0012.

\section{References}

[1] Perkins GD, et al. European resuscitation council guidelines for resuscitation 2015: Section 2. adult basic life support and automated external defibrillation 2015;

[2] Eftest $\varnothing 1$ T, Sunde K, Steen PA. Effects of interrupting precordial compressions on the calculated probability of defibrillation success during out-of-hospital cardiac arrest. Circulation 2002;105(19):2270-2273.

[3] Ruiz J, Ayala U, de Gauna SR, Irusta U, González-Otero
D, Alonso E, Kramer-Johansen J, Eftestøl T. Feasibility of automated rhythm assessment in chest compression pauses during cardiopulmonary resuscitation. Resuscitation 2013; 84(9):1223-1228.

[4] Ayala U, Irusta U, Ruiz J, de Gauna SR, González-Otero D, Alonso E, Kramer-Johansen J, Naas H, Eftest $\varnothing l$ T. Fully automatic rhythm analysis during chest compression pauses. Resuscitation 2015;89:25-30.

[5] Irusta U, Ruiz J, de Gauna SR, Eftestøl T, Kramer-Johansen J. A least mean-square filter for the estimation of the cardiopulmonary resuscitation artifact based on the frequency of the compressions. IEEE Transactions on Biomedical Engineering 2009;56(4):1052-1062.

[6] Gong Y, Gao P, Wei L, Dai C, Zhang L, Li Y. An enhanced adaptive filtering method for suppressing cardiopulmonary resuscitation artifact. IEEE Transactions on Biomedical Engineering 2016;

[7] Aramendi E, Irusta U, Ayala U, Naas H, Kramer-Johansen J, Eftest $\varnothing 1$ T. Filtering mechanical chest compression artefacts from out-of-hospital cardiac arrest data. Resuscitation 2016;98:41-47.

[8] Sysel P, Rajmic P. Goertzel algorithm generalized to non-integer multiples of fundamental frequency. EURASIP Journal on Advances in Signal Processing 2012;2012(1):56.

[9] Xiao Y, Ma L, Ward RK. Fast RLS fourier analyzers capable of accommodating frequency mismatch. Signal Processing 2007;87(9):2197-2212.

[10] Irusta U, Ruiz J, Aramendi E, de Gauna SR, Ayala U, Alonso E. A high-temporal resolution algorithm to discriminate shockable from nonshockable rhythms in adults and children. Resuscitation 2012;83(9):1090-1097.

Address for correspondence:

Name: Iraia Isasi Liñero

Full postal address: Alda Urquijo s/n, 48013,Bilbao,Spain

E-mail address: iraia.isasi@ehu.eus 